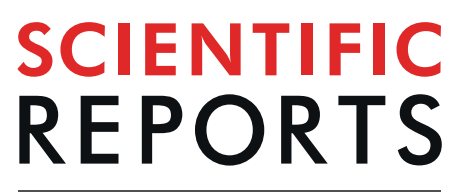

natureresearch

\title{
Three-layer heterogeneous mammographic phantoms for Monte Carlo simulation of normalized glandular dose coefficients in mammography
}

\begin{abstract}
Tien-Yu Chang ${ }^{1}$, Kuan-Jen Lai ${ }^{2}$, Chun-Yuan Tu ${ }^{2,3}$ \& Jay W ${ }^{2 *}$
Normalized glandular dose $(\mathrm{DgN})$ coefficients obtained using homogeneous breast phantoms are commonly used in breast dosimetry for mammography. However, glandular tissue is heterogeneously distributed in the breast. This study aimed to construct three-layer heterogeneous mammographic phantoms (THEPs) to examine the effect of glandular distribution on $\mathrm{DgN}$ coefficient. Each layer of THEPs was set to $25 \%, 50 \%$, or $75 \%$ glandular fraction to emulate heterogeneous glandular distribution. Monte Carlo simulation was performed to attain mean glandular dose (MGD) and air kerma at 22-36 kVp and W/Al, W/Rh, and W/Ag target-filter combinations. The heterogeneous DgN coefficient was calculated as functions of the mean glandular fraction (MGF), breast thickness, tube voltage, and half-value layer. At 50\% MGF, the heterogeneous $\mathrm{DgN}$ coefficients for W/AI, W/Rh, and W/Ag differed by $40.3 \%, 36.7 \%$, and $31.2 \%$. At $9-\mathrm{cm}$ breast thickness, the $\mathrm{DgN}$ values of superior and inferior glandular distributions were $25.4 \%$ higher and $29.2 \%$ lower than those of uniform distribution. The proposed THEPs can be integrated with conventional breast dosimetry to consider the heterogeneous glandular distribution in clinical practice.
\end{abstract}

Breast cancer is the second most common cause of cancer deaths among women ${ }^{1}$, and its incidence increases annually. X-ray mammography has become a primary tool for breast cancer screening because of the high sensitivity and specificity for microcalcification and mass detection ${ }^{2}$. Mammography is also adopted for the high effectiveness/cost ratio. However, the glandular tissue in the breast is sensitive to radiation. The radiation exposure during mammography may increase the risk of radiation-induced secondary breast malignancy ${ }^{3}$. Therefore, assessment of glandular dose in the breast is crucial.

In modern breast dosimetry, one of the most critical parameters is the normalized glandular dose (DgN) coefficient, which is obtained using Monte Carlo simulation of mean glandular dose (MGD) in a breast phantom ${ }^{4}$. Boone used simple homogeneous breast phantoms to simulate the breast absorbed dose and applied a $G$ factor to calculate $\mathrm{MGD}^{5}$. He further proposed monoenergetic $\mathrm{DgN}$ coefficients to facilitate rapid calculation of the DgN coefficient for arbitrary X-ray energy spectra ${ }^{6}$. Dance et al..$^{7}$ employed homogeneous semicylindrical phantoms to assess MGD conversion factor $g$, breast composition factor $c$, and X-ray spectrum factor $s$. The authors further supplemented the conversion factors for the high-energy spectra used for contrast enhanced digital mammography by using the same homogeneous breast model ${ }^{8}$. The above method is now the standard breast dosimetry protocols for mammography in the United Kingdom, European Union, and IAEA and is also adopted in the quality assurance protocols in USA. Sarno et al. ${ }^{9}$ used homogeneous semi-cylinder and ad hoc shaped phantoms to exam MGD in mammography and calculate the t-factor (relative glandular dose, RGD) and T-factor in digital breast tomosynthesis (DBT).

Three-dimensional (3D) imaging modalities, including computed tomography $(\mathrm{CT})^{10,11}$, breast CT (bCT) ${ }^{12,13}$, and magnetic resonance imaging $(\mathrm{MRI})^{14}$, have been used to construct high-resolution breast voxel phantoms

${ }^{1}$ Department of Radiology, Cheng Hsin General Hospital, Taipei, Taiwan. ${ }^{2}$ Department of Biomedical Imaging and Radiological Sciences, National Yang-Ming University, Taipei, Taiwan. ${ }^{3}$ Department of Radiology, Mackay Memorial Hospital, Taipei, Taiwan. *email: jaywu@gm.ym.edu.tw 
(a)

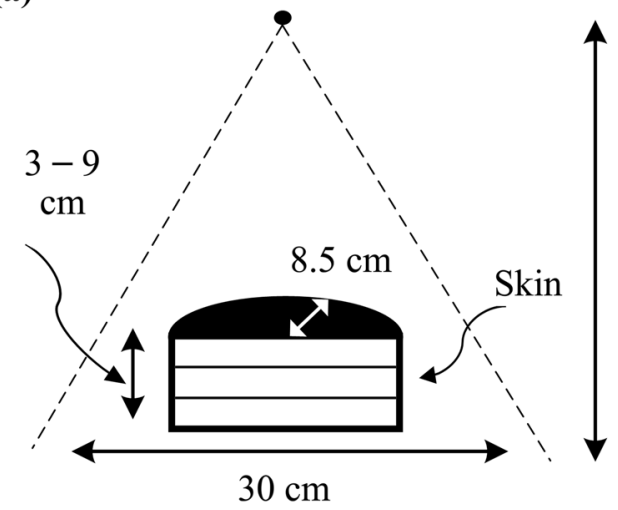

(b)

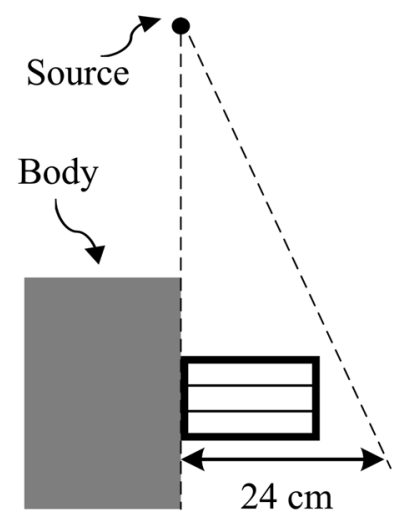

Figure 1. (a) Three-layer heterogeneous mammographic phantom in the form of a semicylinder with a radius of $8.5 \mathrm{~cm}$ and an external skin layer of $0.4 \mathrm{~cm}$, and $(\mathbf{b})$ the geometry of mammography with a THEP and a torso phantom.

to examine the effect of glandular distribution on $\mathrm{DgN}$ coefficient. The results revealed $24 \%$ to $43 \%$ differences in the $\mathrm{DgN}$ values between voxel phantoms and homogeneous phantoms ${ }^{10,12}$. Wang et al. ${ }^{15}$ used breast tissue information adopted from Chinese females to build a series of 3D detailed breast models, which include the retromammary fat, laciferous duct and lobule, intraglandular fat, glandular tissue, subcutaneous fat, Cooper's ligament, and skin. The DgN values of the 3D models were $5.4 \%-38.0 \%$ lower than those of the homogeneous model. Sarno et al. ${ }^{16}$ compared four types of homogeneous breast models with 20 patient-specific digital breast phantoms. The MGD differences were varied from $43 \%$ to $-28 \%$, inferring the importance of glandular distribution in the breast models.

The heterogeneous breast phantoms are usually constructed based on 3D medical images of specific subjects, who cannot represent the entire population. In addition, complete heterogeneous $\mathrm{DgN}$ data sets for various tube voltages, target-filter combinations, beam qualities, breast thicknesses, and glandular distributions are still scarce for MGD and diagnostic reference level (DRL) assessment ${ }^{17}$. This study proposed digital three-layer heterogeneous mammographic phantoms (THEPs) and used the Monte Carlo method to obtain DgN coefficients for different target-filter combinations in mammography. THEPs possess the characteristic of heterogeneous glandular distribution, and the corresponding $\mathrm{DgN}$ coefficients can be integrated into the existing breast dosimetric systems.

\section{Materials and Methods}

Monte carlo simulation. The geometric models of mammography and THEPs were constructed using Monte Carlo N-Particles (MCNP) transport code (version 6.1). Particle tracking was performed using the photon mode, including the following interactions: coherent scattering, photoelectric effect, and Compton scattering. The photon energy cutoff was set to $5 \mathrm{keV}$. Due to the short range of secondary electrons produced in mammography, the kinetic energy of electrons was considered to be locally deposited ${ }^{18}$. Fifty million photons were simulated in each case to reduce the coefficient of variation to less than $2 \%$.

THEP modelling. The THEP was a semicylinder with a radius of $8.5 \mathrm{~cm}$, thicknesses of $3-9 \mathrm{~cm}$, and a skin layer of $0.4 \mathrm{~cm}$ (Fig. 1). It was constructed to emulate the compressed breast in mammography. The inner compartment of THEP was evenly divided into three layers; the glandular fraction (GF) in each layer can be set to $25 \%, 50 \%$, or $75 \%$. When the GF of all three layers is identical, the THEP is consistent with the phantom used in conventional breast dosimetry of mammography ${ }^{6}$ and is referred to as the three-layer homogeneous mammographic phantom (THOP).

Heterogeneous glandular distributions were simulated through the GF combinations of the three layers. For example, at $50 \%$ mean glandular fraction (MGF), the following distributions are possible: uniform distribution (50\%:50\%:50\%), concentrated distribution (25\%:75\%:50\%), inferior distribution (25\%:50\%:75\%), and superior distribution (75\%:50\%:25\%). The elemental composition and density of the tissue with $25 \%, 50 \%$, and $75 \%$ GF as well as the skin were derived from the information of the glandular tissue, adipose tissue, and skin proposed by Hammerstein et al. ${ }^{19}$ (Table 1).

Mammography modelling. The focal spot of the X-ray tube in mammography was set as an isotropic point source, which was $65 \mathrm{~cm}$ away from an image receptor. The THEP was placed at $1.2 \mathrm{~cm}$ above the image receptor, and a $30 \times 20 \times 40 \mathrm{~cm}^{3}$ torso phantom was placed behind the THEP (Fig. 1b). The material of the torso phantom was taken from the soft tissue in the ICRU-44 report ${ }^{20}$. The compression paddle and support plate were not simulated. The source term was generated by the Tungsten Anode Spectral Model using Interpolating Polynomials (TASMIP) software to produce $22-36 \mathrm{kVp}$ tungsten spectra with rhodium, silver, and aluminium filters, respectively ${ }^{21}$. The half-value layers (HVLs) of the energy spectra for commonly used tube voltages are displayed in Table 2. 


\begin{tabular}{|l|l|l|l|l|l|}
\hline & & \multicolumn{5}{|l|}{ Composition (weight percent, \%) } \\
\cline { 3 - 6 } Tissue & Density $\left(\mathbf{g} / \mathbf{c m}^{3}\right)$ & H & C & N & O \\
\hline 25\% GF tissue & 0.955 & 11.0 & 51.0 & 2.1 & 35.7 \\
\hline 50\% GF tissue & 0.982 & 10.7 & 40.1 & 2.5 & 46.4 \\
\hline 75\% GF tissue & 1.010 & 10.5 & 29.3 & 2.9 & 57.0 \\
\hline Skin & 1.090 & 9.8 & 17.8 & 5.0 & 66.7 \\
\hline
\end{tabular}

Table 1. Elemental composition and density of the tissue with $25 \%, 50 \%$, and $75 \%$ GF as well as the skin.

\begin{tabular}{|l|l|l|l|}
\hline $\begin{array}{l}\text { Tube voltage } \\
(\mathbf{k V p})\end{array}$ & $\begin{array}{l}\text { Target-filter } \\
\text { combinations }\end{array}$ & $\begin{array}{l}\text { Filter thickness } \\
(\boldsymbol{\mu} \mathbf{m})\end{array}$ & HVL $(\mathbf{m m ~ A l )}$ \\
\hline 26 & $\mathrm{~W} / \mathrm{Rh}$ & $50,65,80,95$ & $0.460,0.524,0.578,0.622$ \\
\hline 26 & $\mathrm{~W} / \mathrm{Ag}$ & $50,65,80,95$ & $0.471,0.553,0.614,0.667$ \\
\hline 26 & $\mathrm{~W} / \mathrm{Al}$ & $500,650,750,850$ & $0.302,0.377,0.423,0.465$ \\
\hline 30 & $\mathrm{~W} / \mathrm{Rh}$ & $50,65,80,95$ & $0.499,0.563,0.613,0.655$ \\
\hline 30 & $\mathrm{~W} / \mathrm{Ag}$ & $50,65,80,95$ & $0.541,0.620,0.684,0.740$ \\
\hline 30 & $\mathrm{~W} / \mathrm{Al}$ & $500,650,750,850$ & $0.356,0.440,0.499,0.549$ \\
\hline 36 & $\mathrm{~W} / \mathrm{Rh}$ & $50,65,80,95$ & $0.548,0.608,0.652,0.690$ \\
\hline 36 & $\mathrm{~W} / \mathrm{Ag}$ & $50,65,80,95$ & $0.606,0.679,0.740,0.792$ \\
\hline 36 & $\mathrm{~W} / \mathrm{Al}$ & $500,650,750,850$ & $0.434,0.528,0.600,0.661$ \\
\hline
\end{tabular}

Table 2. Half-value layers of the energy spectra for commonly used exposure parameters in mammography.

MGD and air kerma simulation. Photons were simulated from the focal spot to the image receptor, and the energy deposited in each layer of the THEP was recorded. The MGD was calculated as follows:

$$
\begin{gathered}
M G D=\frac{\sum_{i} G_{i}(E) \times E_{i}}{\sum_{i} V \times \rho_{i} \times f_{\mathrm{g}, i}} \\
G(E)=\frac{f_{\mathrm{g}} \times\left(\frac{\mu_{\mathrm{en}}}{\rho}\right)_{\mathrm{g}}}{f_{\mathrm{g}} \times\left(\frac{\mu_{\mathrm{en}}}{\rho}\right)_{\mathrm{g}}+\left(1-f_{\mathrm{g}}\right) \times\left(\frac{\mu_{\mathrm{en}}}{\rho}\right)_{\mathrm{a}}}
\end{gathered}
$$

where $E_{i}$ is the energy deposited in layer $i, V$ is the volume of each layer, and $\rho_{i}$ is the tissue density in layer $i$. $G(E)$ is the energy absorption ratio of the glandular tissue to total breast calculated by the mass energy absorption coefficients of glandular tissue $\left(\mu_{\mathrm{en}} / \rho\right)_{\mathrm{g}}$ and adipose tissue $\left(\mu_{e n} / \rho\right)_{a} ; f_{g}$ and $\left(1-f_{g}\right)$ are the weight percentages of glandular and adipose tissues, respectively. In addition, we constructed the geometric model of a pancake ionisation chamber with a radius of $14.5 \mathrm{~mm}$ and thickness of $2 \mathrm{~mm}$ to simulate air kerma in the active volume. The chamber was filled with dry air and placed at the position of the THEP. The breast and torso phantoms were removed to avoid backscattered photons. The compression paddle was not simulated as well to avoid large angular spread of X-ray photons on the detector surface ${ }^{22}$.

DgN calculation. Twenty-seven glandular distributions were generated from different combinations of GF in each layer of the THEP. The DgN coefficient was calculated by dividing the MGD by the air kerma for different $\mathrm{X}$-ray energy spectra and $3-9 \mathrm{~cm}$ breast thicknesses, as follows:

$$
D g N=\frac{M G D}{K}
$$

where $K$ is the air kerma. For verifying the Monte Carlo modelling, THOPs with $25 \%, 50 \%$, and $75 \%$ MGF were employed to attain the DgN coefficients at the W/Ag target-filter combination for breast thicknesses of 3, 5, and $7 \mathrm{~cm}$ and tube voltages of 26, 28, 30,32, and $34 \mathrm{kVp}$. The results were compared with those obtained by Nosratieh et al. ${ }^{23}$. Subsequently, THEPs were used to obtain the heterogeneous DgN coefficients for concentrated, inferior, and superior glandular distributions. The relationships between the heterogeneous DgN coefficient, MGF, breast thickness, tube voltage, and HVL were established.

\section{Results}

Validation of Monte Carlo code. We used MCNP 6.1 with the MCPLIB84 cross-section library to simulate the case 3 in the AAPM TG-195 report for mammography dosimetry ${ }^{24}$. The breast model was a semicylinder with a radius of $98 \mathrm{~mm}$, thicknesses of $46 \mathrm{~mm}$, and skin layer of $2 \mathrm{~mm}$. The interior composition of the model was a homogeneous mixture of $80 \%$ adipose tissue and $20 \%$ glandular tissue. A polymethyl-methacrylate 


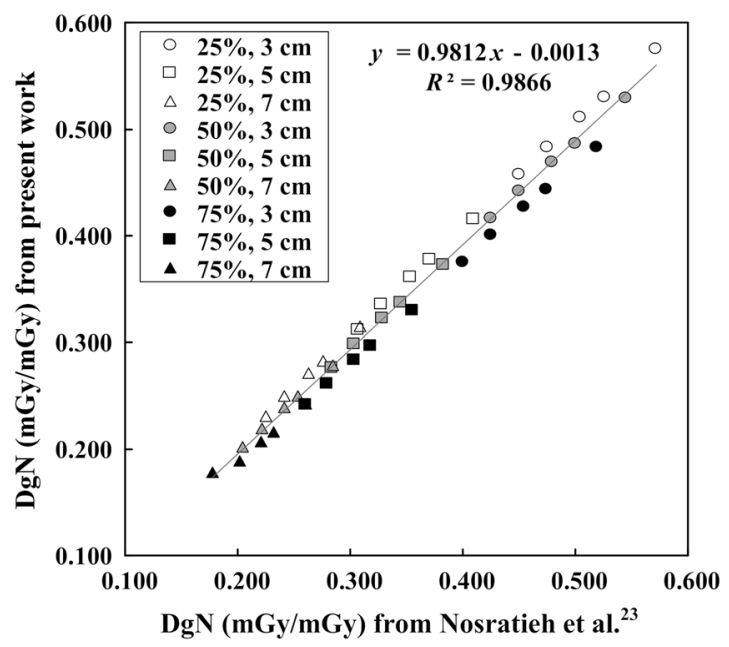

Figure 2. Scatter plot of the DgN coefficients of THOPs and those obtained by Nosratieh et al. ${ }^{23}$. The linear regression result showed a good linearity with an $R^{2}$ of 0.9866 .

rectangular box with a dimension of $140 \times 260 \times 2 \mathrm{~mm}^{3}$ was added above and below the breast model as compression and support paddles. The distance between the source and compression paddle was $59.3 \mathrm{~cm}$, and photons with $16.8 \mathrm{keV}$ were emitted from the source isotropically. The result of breast energy deposition per photon was $4707 \mathrm{eV} /$ photon $(\mathrm{CV}=0.42 \%)$. Compared with the result of TG-195, the error is less than $0.2 \%$, indicating the credibility of the Monte Carlo code.

Verification of DgN coefficients. Figure 2 compares the DgN coefficients of THOPs and those obtained by Nosratieh et al. ${ }^{23}$. The linear regression result revealed a favorable linearity $\left(y=0.9812 x-0.0013, R^{2}=0.9866\right)$ with no significant differences between the two sets of $\mathrm{DgN}$ values $(p=0.727)$. The mean $\mathrm{DgN}$ differences at $25 \%$ and $50 \%$ MGF were $2.2 \%$ and $1.8 \%$, respectively, whereas the difference slightly increased to $6.4 \%$ at $75 \%$ MGF. By using the same X-ray spectral model proposed by Boone et al. ${ }^{21}$, Sarno et al..$^{25}$ also compared their simulation data with those proposed by Nosratieh et al., and showed that $5 \%$ higher DgN coefficients on average. This result infers that the DgN coefficients of THOPs may be approximately 7\% lower that the results obtained by Sarno et al.

Heterogeneous DgN coefficient and mean glandular fraction. Figure 3 illustrates the heterogeneous $\mathrm{DgN}$ coefficients of THEPs as a function of MGF for three commonly used exposure conditions, (1) $26 \mathrm{kVp}, \mathrm{Al}$ filter, and 0.423-mm HVL, (2) $30 \mathrm{kVp}$, Rh filter, and 0.563-mm HVL, and (3) $36 \mathrm{kVp}$, Ag filter, and 0.679-mm HVL. The DgN coefficient had an inverse trend with MGF and breast thickness. The scattered data points for a given breast thickness and MGF represent the heterogeneous $\mathrm{DgN}$ coefficients resulting from different glandular distributions. At 4-cm breast thickness and 50\% MGF, the differences in the heterogeneous DgN coefficients for the $\mathrm{W} / \mathrm{Al}, \mathrm{W} / \mathrm{Rh}$, and $\mathrm{W} / \mathrm{Ag}$ target-filter combinations were $40.3 \%, 36.7 \%$, and $31.2 \%$, respectively.

Heterogeneous DgN coefficient and breast thickness. Figure 4 depicts the heterogeneous DgN coefficients of THEPs for breast thicknesses of 3 to $9 \mathrm{~cm}$ for the three commonly used exposure conditions at $50 \%$ MGF. The DgN coefficient decreased with an increase in breast thickness. In the situation of superior glandular distribution, the $\mathrm{DgN}$ value was higher than that of uniform distribution; conversely, the DgN value of inferior distribution was lower than that of uniform distribution. At $4-\mathrm{cm}$ breast thickness, the DgN coefficient of the upper concentrated distribution (50\%:75\%:25\%) was higher than that of the lower concentrated distribution (25\%:75\%:50\%) by 19.3\%, 17.6\%, and 15.1\% for W/Al, W/Rh, and W/Ag target-filter combinations.

Heterogeneous DgN coefficient and tube voltage. Figure 5 shows the heterogeneous DgN coefficients of THEPs as a function of tube voltage at W/Ag target-filter combination, $0.658-\mathrm{mm} \mathrm{HVL}$, and 50\% MGF. The $\mathrm{DgN}$ coefficient was positively correlated to tube voltage but negatively correlated to breast thickness. At $26 \mathrm{kVp}$, the mean $\mathrm{DgN}$ coefficient of inferior glandular distribution of small breasts $(3-6 \mathrm{~cm})$ was $16.8 \%$ lower than that of uniform distribution, whereas the mean $\mathrm{DgN}$ value of superior distribution was $15.3 \%$ higher than that of uniform distribution. When the tube voltage increased to $34 \mathrm{kVp}$, the mean $\mathrm{DgN}$ coefficients of inferior and superior distributions were $15.9 \%$ lower and $14.6 \%$ higher than that of uniform distribution, respectively. The results suggest that the effect of glandular distribution on $\mathrm{DgN}$ coefficient slightly decreases with increasing tube voltage.

Heterogeneous DgN coefficient and HVL. Figure 6 shows the heterogeneous DgN coefficients of THEPs for various HVLs at $32 \mathrm{kVp}$, W/Al target-filter combination, and 50\% MGF. The $\mathrm{DgN}$ value was positively correlated with HVL. At 0.469-mm HVL and 4-cm breast thickness, the DgN coefficient of inferior glandular distribution was $15.0 \%$ lower than that of uniform distribution, whereas the DgN coefficient of superior distribution was 13.9\% higher than that of uniform distribution. At the same HVL but 9-cm breast thickness, the DgN coefficients 


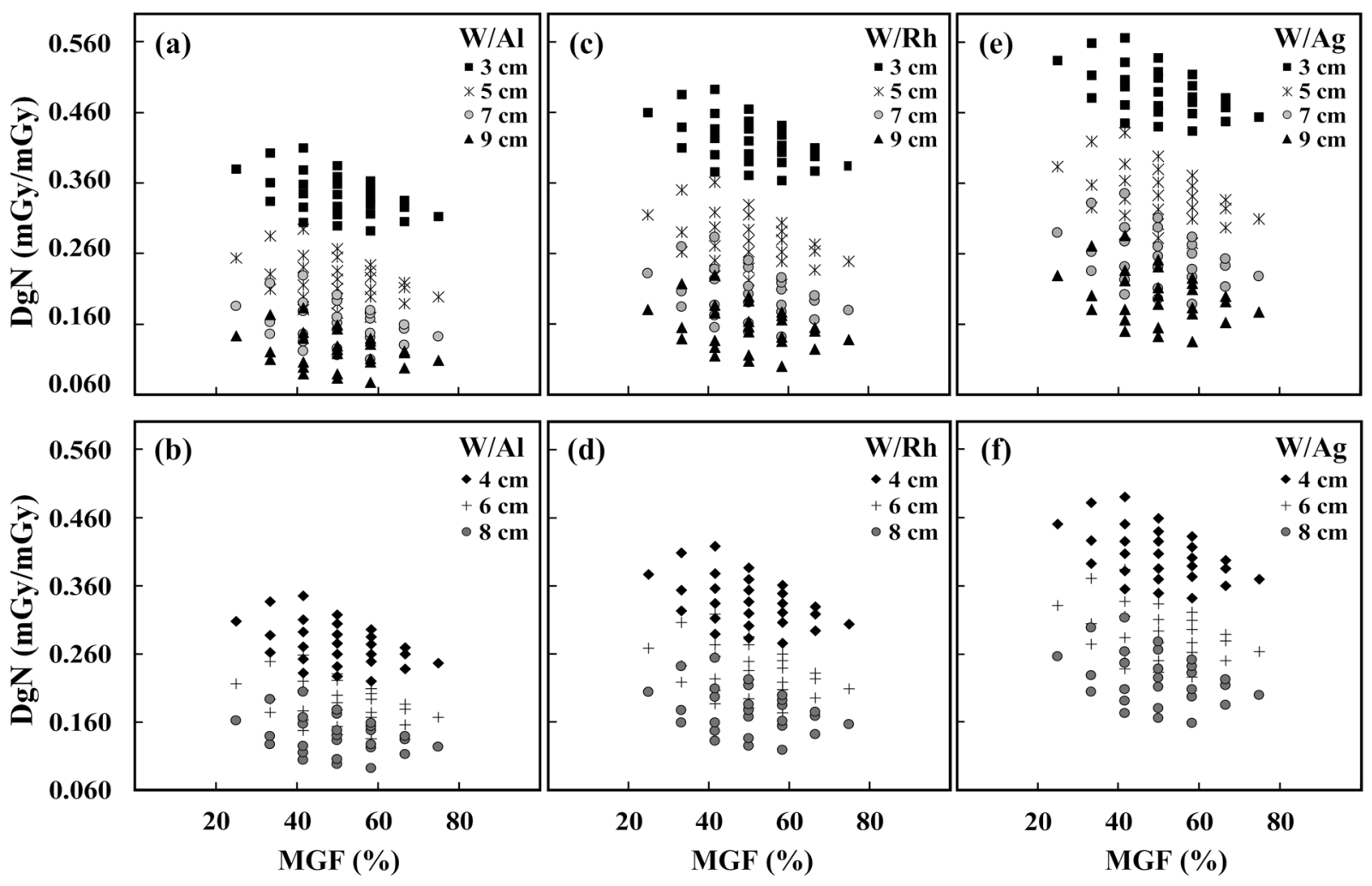

Figure 3. Heterogeneous $\mathrm{DgN}$ coefficient versus mean glandular fraction for three commonly used exposure conditions: (a)(b) $26 \mathrm{kVp}$, Al filter, and 0.423-mm HVL, (c)(d) $30 \mathrm{kVp}$, Rh filter, and 0.563-mm HVL, and (e)(f) $36 \mathrm{kVp}, \mathrm{Ag}$ filter, and 0.679-mm HVL. Upper row and lower row represent breast thicknesses of 3, 5, 7, and $9 \mathrm{~cm}$ and breast thicknesses of 4,6 , and $8 \mathrm{~cm}$, respectively.

of inferior and superior distributions were $29.2 \%$ lower and $25.4 \%$ higher than that of uniform distribution. The glandular distribution has a stronger impact on $\mathrm{DgN}$ coefficient in larger breasts.

Heterogeneous DgN coefficient for clinical exposure conditions. Table 3 lists clinical exposure parameters and heterogeneous DgN coefficients for different glandular distributions of small, medium, and large breasts at $50 \%$ MGF. At the breast thicknesses of 4,6 , and $8 \mathrm{~cm}$, the DgN coefficients of superior distribution were $15.6 \%, 21.3 \%$, and $23.0 \%$ higher than that of uniform distribution, respectively; in contrast, the DgN values of inferior distribution were $17.8 \%, 23.8 \%$, and $26.5 \%$ lower than that of uniform distribution. The results again reveal that breast thickness has a strong influence of glandular distribution on DgN coefficient.

\section{Discussion}

This study showed that the DgN coefficient is negatively correlated with breast thickness, which is consistent with the earlier investigation by Wu et al. ${ }^{26}$ and Santos et al. ${ }^{27}$. The primary reason for this trend is that the breast mass increases with increasing breast thickness, thus lowering the MGD and the corresponding DgN coefficient. The $\mathrm{DgN}$ coefficient also has an inverse relationship with MGF, which is mainly because the tissue with higher GF attenuates more $\mathrm{X}$-ray photons and lowers the percent depth dose.

At 50\% MGF, seven glandular distributions can be generated in THEPs, mainly uniform (50\%:50\%:50\%), inferior (25\%:50\%:75\%), superior (75\%:50\%:25\%), lower concentrated (25\%:75\%:50\%), and upper concentrated (50\%:75\%: 25\%) distributions. The remaining two, 50\%:25\%:75\% and 75\%:25\%:50\%, are relatively uncommon in clinical practice. It is worth mentioning that the uniform distribution is the one used in the conventional mammographic dosimetry. The glandular distribution affects the $\mathrm{DgN}$ coefficient substantially. When the GF of the first layer of THEPs increases, the $\mathrm{DgN}$ value increases. This trend also applies to the upper concentrated glandular distribution, which has a higher $\mathrm{DgN}$ value than the lower concentrated distribution. These results can be explained by the exponential attenuation of X-ray photons that deposit most of their energy near the photon entrance side of the breast. Higher GF in the first layer thus substantially contributes to the MGD. In clinical practice, inferior and lower concentrated glandular distributions are more common than other types of distributions, indicating the use of homogeneous DgN coefficient may result in overestimation of MGD.

Higher tube voltage and HVL are often used for large breasts in mammography. As tube voltage increases, more energy is deposited in the breast regardless of the glandular distribution, resulting in a higher MGD and $\mathrm{DgN}$ coefficient. However, at high $\mathrm{kVp}$, the effect of glandular distribution on DgN coefficient slightly decreases because the difference in the mass energy absorption coefficient between adipose and glandular tissues decreases. For reducing skin dose, additional filters are added to attenuate low-energy photons and consequently elevate the 
(a)

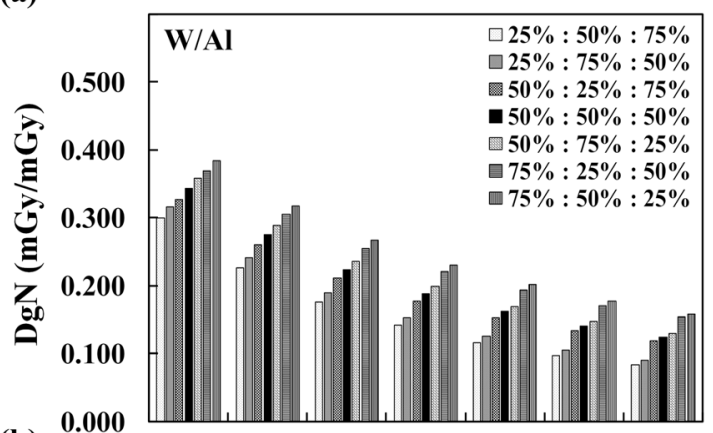

(b)
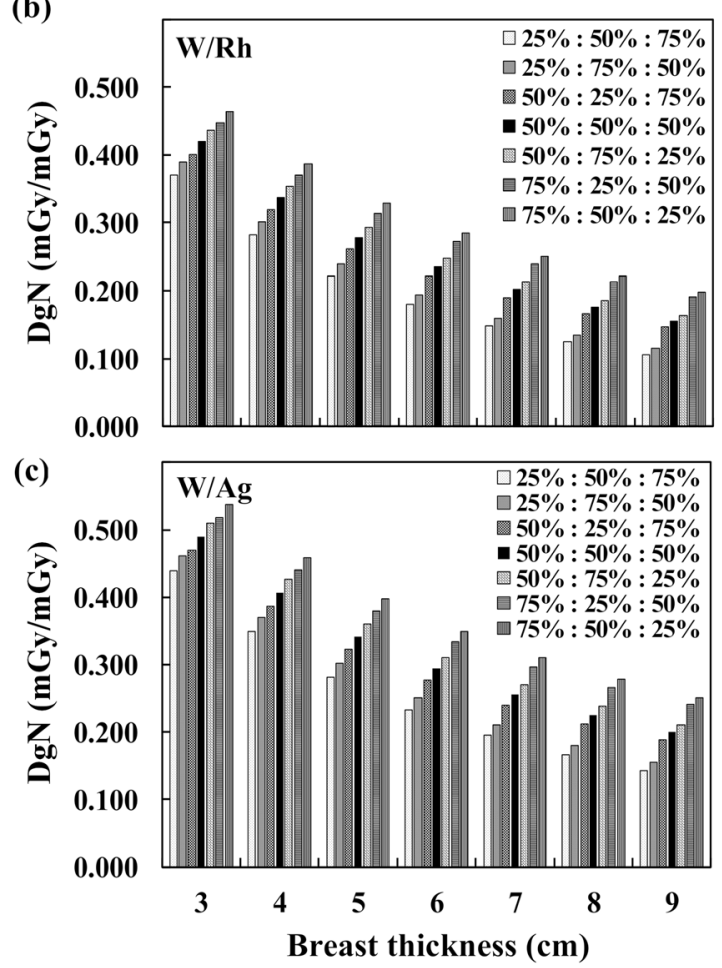

Figure 4. Heterogeneous DgN coefficient versus breast thickness for the three commonly used exposure parameters of (a) $26 \mathrm{kVp}$, W/Al, and $0.423-\mathrm{mm} \mathrm{HVL}$, (b) $30 \mathrm{kVp}, \mathrm{W} / \mathrm{Rh}$, and $0.563-\mathrm{mm} \mathrm{HVL}$, and (c) $36 \mathrm{kVp}$, $\mathrm{W} / \mathrm{Ag}$, and 0.679-mm HVL.

(a)

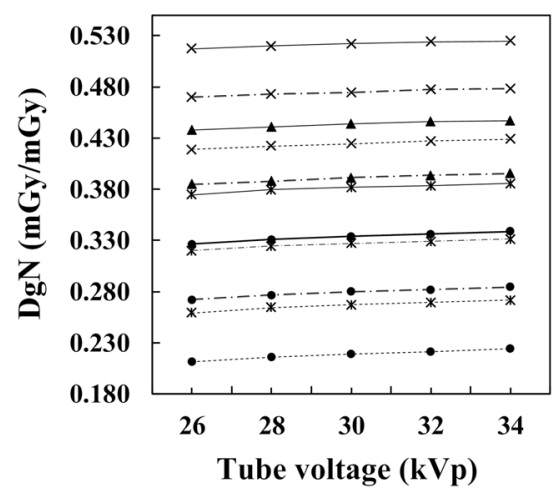

(b)

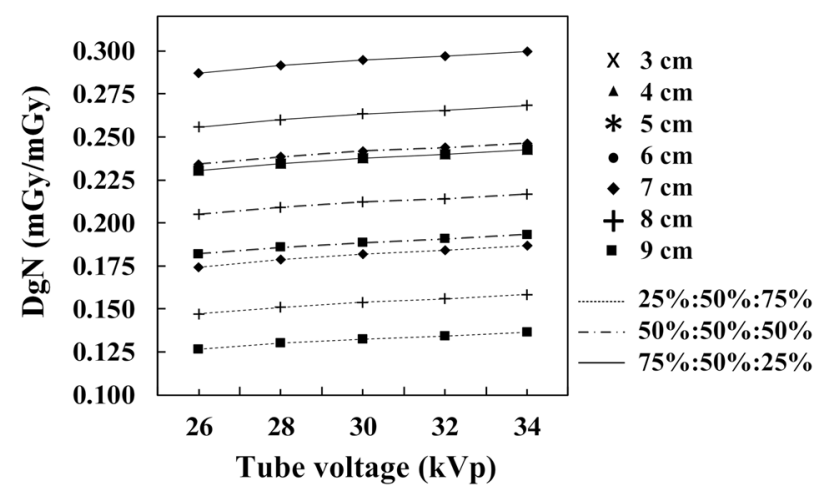

Figure 5. Heterogeneous DgN coefficient versus tube voltage for (a) 3 to $6-\mathrm{cm}$ small breasts and (b) 7 to $9-\mathrm{cm}$ large breasts at W/Ag target-filter combination, 0.658-mm HVL, and 50\% MGF. The DgN coefficient was positively correlated with tube voltage. 
(a)

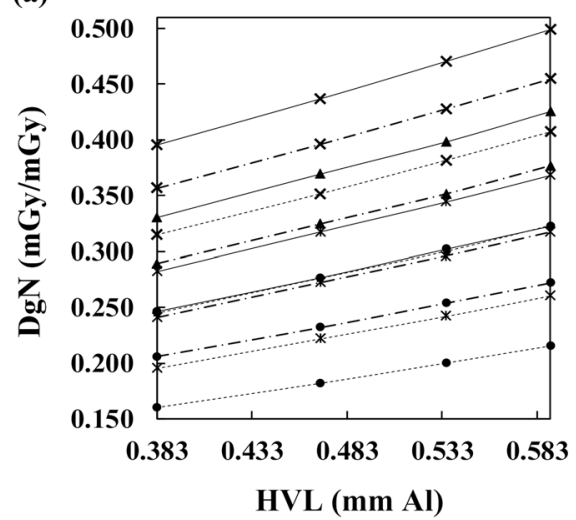

(b)

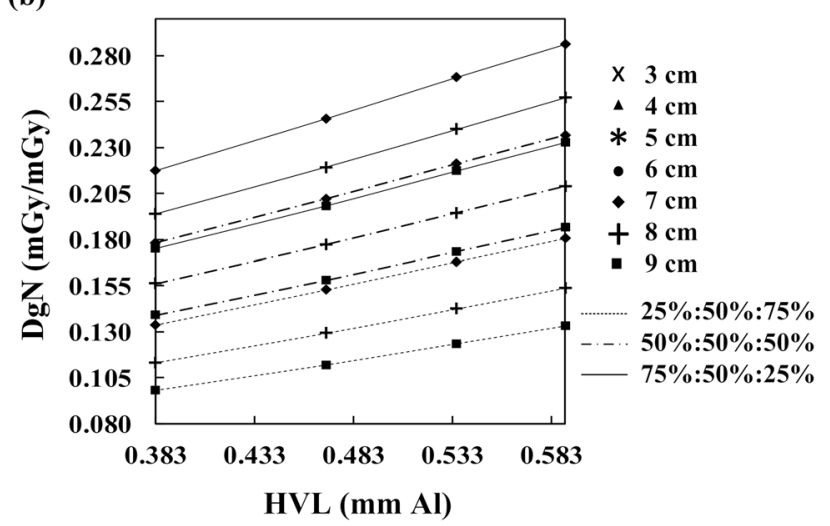

Figure 6. Heterogeneous DgN coefficient versus HVL for (a) 3 to 6 -cm small breasts and (b) 7 to 9-cm large breasts at $32 \mathrm{kVp}$, W/Al target-filter combination, and 50\% MGF. The DgN coefficient was positively correlated with HVL.

\begin{tabular}{|c|c|c|c|c|c|c|}
\hline \multirow{2}{*}{$\begin{array}{l}\text { Tube voltage } \\
(\mathbf{k V p})\end{array}$} & \multirow{2}{*}{$\begin{array}{l}\text { Target-filter } \\
\text { combination }\end{array}$} & \multirow[b]{2}{*}{ HVL (mm Al) } & \multirow{2}{*}{$\begin{array}{l}\text { CBT } \\
(\mathrm{cm})\end{array}$} & \multicolumn{3}{|c|}{$\mathrm{DgN}$ (mGy/mGy) } \\
\hline & & & & superior & uniform & inferior \\
\hline 26 & W/Al & 0.423 & 4 & 0.318 & 0.275 & 0.226 \\
\hline 30 & $\mathrm{~W} / \mathrm{Rh}$ & 0.563 & 6 & 0.285 & 0.235 & 0.179 \\
\hline 36 & W/Ag & 0.679 & 8 & 0.278 & 0.226 & 0.166 \\
\hline
\end{tabular}

Table 3. Clinical exposure parameters and heterogeneous $\mathrm{DgN}$ coefficients for superior, inferior, and uniform distributions of small, medium, and large breasts.

HVL of X-ray spectra. Similarly, the DgN coefficient increases and the effect of glandular distribution on DgN coefficient decreases because of the increase in the average energy of X-ray.

Hernandez et al. ${ }^{12}$ used bCT images to construct heterogeneous phantoms, indicating the DgN coefficient can vary by $-22.1 \%$ to $28.5 \%$. The results of the present study revealed a similar trend. Additionally, we showed that glandular distribution has a stronger effect on $\mathrm{DgN}$ coefficient for larger breasts. The DgN value varies from $-29.2 \%$ to $25.4 \%$ at $9-\mathrm{cm}$ breast thickness, whereas the $\mathrm{DgN}$ differences are from $-15.0 \%$ to $13.9 \%$ in medium-sized breasts. These findings conclude that using the DgN coefficient obtained from simple homogeneous phantoms may cause a $30 \%$ error in MGD evaluation.

In clinical practice, the MGF can be determined using Breast Imaging Reporting and Data System (BI-RADS) or other clinically available software through mammogram analysis, such as the Laboratory for Individualized Breast Radiodensity Assessment (LIBRA) ${ }^{28}$ and Volpara ${ }^{29}$. The glandular distribution pattern can be evaluated from the mediolateral view of mammograms by drawing regions of interest (ROIs) in the upper, middle, and lower regions inside the breast, respective. The grayscale ratio between the ROIs is used as the basis for breast distribution. Other 3D imaging modalities, such as DBT, bCT, and MRI, are also suitable for estimating the GF ratio in each layer of THEPs. By using the threshold method, the GF in each layer can be calculated as the number of glandular pixels divided by the number of whole breast pixels in that layer.

There are various sources of systematic errors in the calculation of DgN coefficients. Firstly, in the elemental composition and density of materials, several data sources, including Hammerstein's ${ }^{19}$ and ICRU $44^{20}$, show a wide variability for both adipose and glandular tissues. Chen et al. ${ }^{30}$ compared the linear attenuation coefficients of adipose and glandular tissues taken from different studies and showed that the results of glandular tissue from the Hammerstein's data are in good agreement with those from the other three studies ${ }^{30-32}$. However, at lower energies, the linear attenuation coefficients of adipose tissue are slightly inconsistent among the three studies, which inevitably increases the uncertainty of MGD simulation. To further investigate this uncertainty, we used the data from ICRU 44 instead of Hammerstein's data in THOPs, and the results shows approximately $3 \%$ uncertainty in MGD. Secondly, oversimplified breast models have been used for different clinical breast dose assessment protocols, which overestimate MGD by about $30 \%{ }^{12}$. If the purpose of MGD evaluation is to reflect the average population breast dose for radiation protection and DRL assessment, mildly conservative overestimation suits the basic principles of radiation protection ${ }^{33}$. Finally, the systematic errors may come from the Monte Carlo code and its cross-section library. Our simulation result of the case 3 in the AAPM TG-195 report for mammography dosimetry has a difference less than $0.2 \%$ compared with the result of TG-195. Different Monte Carlo programs, including EGSnrc, Geant4, MCNPX, and Penelope, may also cause $0.2 \%$ uncertainty in MGD evaluation ${ }^{24}$.

The purpose of using heterogeneous phantoms derived from clinical CT scans for MGD simulations is to overcome the problem of non-uniform distribution of breast tissue. New clinical breast dosimetric systems should be developed from this perspective. However, existing clinical systems still use simple homogenous phantoms in their glandular dose assessment ${ }^{34}$ : Philips uses Dance's system, GE uses Wu's system, and Hologic uses Boone's 
system. Our proposed THEPs are compatible with the simple phantom used in clinical dosimetric systems and are intended to correct the problem of overestimated MGD originating from homogeneous breast tissue assumption. For example, the glandular tissue in breasts is often inferiorly distributed. By using the THEP protocol, the dose can be reduced by $17 \%$ to $26 \%$ according to the results listed in Table 3 . Before the DgN coefficients obtained from those heterogeneous and voxel phantoms find a widespread use in digital mammography systems, correction of the $\mathrm{DgN}$ results can be obtained with the help of THEP.

The thickness of the skin layer has a crucial influence on MGD assessment. Dance ${ }^{35}$ and Boone $e^{5}$ employed the breast models with 5- and 4-mm-thick skin layers in the currently used dosimetric systems, respectively. However, by using bCT images, Huang et al. ${ }^{36}$ and Vedantham et al. ${ }^{37}$ analysed the breast skin thickness to be $1.45 \mathrm{~mm}$. The thick skin layer attenuates more X-ray photons, thereby reducing the MGD by up to $27 \%{ }^{18,38}$. To be compatible with these clinically used breast dosimetric systems, the skin thickness of THEPs was set to $4 \mathrm{~mm}$. Although the calculated DgN coefficients may indeed be underestimated, the ratio of DgN of different glandular distributions to DgN of uniform glandular distribution remains valid. This ratio can be fed into other breast dose assessment systems to correct for the skin thickness problem, while solving the problem of non-uniform distribution of breast tissue at the same time. Finally, the question of optimising the skin thickness of THEPs merits further research.

\section{Conclusion}

This study employed THEPs to investigate the DgN coefficient for uniform, concentrated, superior, and inferior glandular distributions. The results indicate that $\mathrm{DgN}$ coefficient is negatively correlated to breast thickness and MGF, whereas it is positively correlated to tube voltage and HVL. The effect of heterogeneous glandular distribution on $\mathrm{DgN}$ coefficient is significantly stronger for large breasts, but it is slightly weaker at high photon energy and HVL. The glandular distribution may result in a $29.2 \%$ difference in the DgN coefficient at $50 \%$ MGF. The proposed THEPs and heterogeneous DgN coefficients can consider the breast thickness, mean glandular fraction, glandular distribution, tube voltage, beam quality, and target-filter combination, while facilitating the integration with conventional breast dosimetry to provide clinical MGD and DRL evaluation.

\section{Data availability}

The datasets generated and analysed during the current study are available from the corresponding author on reasonable request.

Received: 9 August 2019; Accepted: 27 January 2020;

Published online: 10 February 2020

\section{References}

1. Siegel, R. L., Miller, K. D. \& Jemal, A. Cancer statistics, 2018. CA: A Cancer J. Clinicians 68, 7-30 (2018).

2. Lehman, C. D. et al. Diagnostic accuracy of digital screening mammography with and without computer-aided detection. JAMA Intern. Med. 175, 1828-1837 (2015).

3. RMK, M. A. et al. Effective lifetime radiation risk for a number of national mammography screening programmes. Radiography $\mathbf{2 4}$, 240-246 (2018)

4. Ciraj-Bjelac, O., Beciric, S., Arandjic, D., Kosutic, D. \& Kovacevic, M. Mammography radiation dose: initial results from Serbia based on mean glandular dose assessment for phantoms and patients. Radiat. Prot. Dosimetry 140, 75-80 (2010).

5. Boone, J. M. Glandular breast dose for monoenergetic and high-energy X-ray beams: Monte Carlo assessment. Radiology 213, 23-37 (1999).

6. Boone, J. M. Normalized glandular dose $(\mathrm{DgN})$ coefficients for arbitrary X-ray spectra in mammography: computer-fit values of Monte Carlo derived data. Med. Phys. 29, 869-875 (2002).

7. Dance, D. R., Skinner, C. L., Young, K. C., Beckett, J. R. \& Kotre, C. J. Additional factors for the estimation of mean glandular breast dose using the UK mammography dosimetry protocol. Phys. Med. Biol. 45, 3225-3240 (2000).

8. Dance, D. R. \& Young, K. C. Estimation of mean glandular dose for contrast enhanced digital mammography: factors for use with the UK, European and IAEA breast dosimetry protocols. Phys. Med. Biol. 59, 2127-2137 (2014).

9. Sarno, A. et al. Normalized glandular dose coefficients in mammography, digital breast tomosynthesis and dedicated breast CT. Phys. Med. 55, 142-148 (2018).

10. Dance, D. R. et al. Breast dosimetry using high-resolution voxel phantoms. Radiat. Prot. Dosimetry 114, 359-363 (2005).

11. O’Connor, J. M., Das, M., Dider, C. S., Mahd, M. \& Glick, S. J. Generation of voxelized breast phantoms from surgical mastectomy specimens. Med. Phys. 40, 041915 (2013).

12. Hernandez, A. M., Seibert, J. A. \& Boone, J. M. Breast dose in mammography is about $30 \%$ lower when realistic heterogeneous glandular distributions are considered. Med. Phys. 42, 6337-6348 (2015).

13. Sturgeon, G. M., Park, S., Segars, W. P. \& Lo, J. Y. Synthetic breast phantoms from patient based eigenbreasts. Med. Phys. 44, 6270-6279 (2017).

14. Garcia, E. et al. A step-by-step review on patient-specific biomechanical finite element models for breast MRI to x-ray mammography registration. Med. Phys. 45, e6-e31 (2018).

15. Wang, W. et al. Monte Carlo calculation of conversion coefficients for dose estimation in mammography based on a 3D detailed breast model. Med. Phys. 44, 2503-2514 (2017).

16. Sarno, A. et al. Homogeneous vs. patient specific breast models for Monte Carlo evaluation of mean glandular dose in mammography. Phys. Med. 51, 56-63 (2018).

17. Suleiman, M. E., McEntee, M. F., Cartwright, L., Diffey, J. \& Brennan, P. C. Diagnostic reference levels for digital mammography in New South Wales. J. Med. Imaging Radiat. Oncol. 61, 48-57 (2017).

18. Sarno, A., Mettivier, G., Di Lillo, F. \& Russo, P. A Monte Carlo study of monoenergetic and polyenergetic normalized glandular dose (DgN) coefficients in mammography. Phys. Med. Biol. 62, 306-325 (2017).

19. Hammerstein, G. R. et al. Absorbed radiation dose in mammography. Radiology 130, 485-491 (1979).

20. Bethesda, M. Report 44: Tissue substitutes in radiation dosimetry and measurement. International Commission on Radiation Units and Measurements (1989)

21. Boone, J. M., Fewell, T. R. \& Jennings, R. J. Molybdenum, rhodium, and tungsten anode spectral models using interpolating polynomials with application to mammography. Med. Phys. 24, 1863-1874 (1997) 
22. Sarno, A., Mettivier, G. \& Russo, P. Air kerma calculation in Monte Carlo simulations for deriving normalized glandular dose coefficients in mammography. Phys. Med. Biol. 62, N337-N349 (2017).

23. Nosratieh, A. et al. Mean glandular dose coefficients $(\mathrm{D}(\mathrm{g}) \mathrm{N})$ for $\mathrm{x}$-ray spectra used in contemporary breast imaging systems. Phys. Med. Biol. 60, 7179-7190 (2015).

24. Sechopoulos, I. et al. Monte Carlo reference data sets for imaging research: Executive summary of the report of AAPM Research Committee Task Group 195. Med. Phys. 42, 5679-5691 (2015).

25. Sarno, A., Tucciariello, R. M., Mettivier, G., di Franco, F. \& Russo, P. Monte Carlo calculation of monoenergetic and polyenergetic $\mathrm{DgN}$ coefficients for mean glandular dose estimates in mammography using a homogeneous breast model. Phys. Med. Biol. 64, 125012 (2019).

26. Wu, X., Gingold, E. L., Barnes, G. T. \& Tucker, D. M. Normalized average glandular dose in molybdenum target-rhodium filter and rhodium target-rhodium filter mammography. Radiology 193, 83-89 (1994).

27. Santos, J. C., Tomal, A., de Barros, N. \& Costa, P. R. Normalized glandular dose (DgN) coefficients from experimental mammographic x-ray spectra. Phys. Med. Biol. 64, 105010 (2019).

28. Lee, J. \& Nishikawa, R. M. Automated mammographic breast density estimation using a fully convolutional network. Med. Phys. 45, $1178-1190$ (2018).

29. Salomon, E. et al. Comparison of a personalized breast dosimetry method with standard dosimetry protocols. Sci. Rep. 9, 5866 (2019).

30. Chen, R. C. et al. Measurement of the linear attenuation coefficients of breast tissues by synchrotron radiation computed tomography. Phys. Med. Biol. 55, 4993-5005 (2010).

31. Johns, P. C. \& Yaffe, M. J. X-ray characterisation of normal and neoplastic breast tissues. Phys. Med. Biol. 32, 675-695 (1987).

32. Tomal, A., Mazarro, I., Kakuno, E. \& Poletti, M. Experimental determination of linear attenuation coefficient of normal, benign and malignant breast tissues. Radiat. Meas. 45, 1055-1059 (2010).

33. ICRP. ICRP publication 103. Ann. ICRP 37, 2 (2007).

34. Suleiman, M. E., Brennan, P. C. \& McEntee, M. F. Mean glandular dose in digital mammography: a dose calculation method comparison. J. Med. Imaging 4, 013502 (2017).

35. Dance, D. R. Monte Carlo calculation of conversion factors for the estimation of mean glandular breast dose. Phys. Med. Biol. 35, $1211-1219(1990)$.

36. Huang, S. Y., Boone, J. M., Yang, K., Kwan, A. L. \& Packard, N. J. The effect of skin thickness determined using breast CT on mammographic dosimetry. Med. Phys. 35, 1199-1206 (2008).

37. Vedantham, S., Shi, L., Karellas, A. \& O'Connell, A. TU-E-217BCD-05: Dedicated Breast CT: Skin Thickness Measurements in a Diagnostic Population. Med. Phys. 39, 3914-3915 (2012).

38. Massera, R. T. \& Tomal, A. Skin models and their impact on mean glandular dose in mammography. Phys. Med. 51, 38-47 (2018).

\section{Acknowledgements}

This study was partly supported by Cheng Hsin general hospital (CY10801) and by Ministry of Science and Technology of Taiwan (MOST 107-2314-B-010-053).

\section{Author contributions}

J.W. and T.-Y.C. designed the experiments. K.-J.L. and C.-Y.T. performed the experiments. J.W. wrote the paper.

\section{Competing interests}

The authors declare no competing interests.

\section{Additional information}

Correspondence and requests for materials should be addressed to J.W.

Reprints and permissions information is available at www.nature.com/reprints.

Publisher's note Springer Nature remains neutral with regard to jurisdictional claims in published maps and institutional affiliations.

Open Access This article is licensed under a Creative Commons Attribution 4.0 International License, which permits use, sharing, adaptation, distribution and reproduction in any medium or format, as long as you give appropriate credit to the original author(s) and the source, provide a link to the Creative Commons license, and indicate if changes were made. The images or other third party material in this article are included in the article's Creative Commons license, unless indicated otherwise in a credit line to the material. If material is not included in the article's Creative Commons license and your intended use is not permitted by statutory regulation or exceeds the permitted use, you will need to obtain permission directly from the copyright holder. To view a copy of this license, visit http://creativecommons.org/licenses/by/4.0/.

(C) The Author(s) 2020 